\title{
Author Correction: Hypericin-mediated sonodynamic therapy induces autophagy and decreases lipids in THP-1 macrophage by promoting ROS-dependent nuclear translocation of TFEB
}

Xuesong Li ${ }^{1}$, Xin Zhang ${ }^{2,3}$, Longbin Zheng ${ }^{1}$, Jiayuan Kou' ${ }^{1}$, Zhaoyu Zhong ${ }^{1}$, Yueqing Jiang ${ }^{1}$, Wei Wang ${ }^{3}$, Zengxiang Dong ${ }^{3}$, Zhongni Liu', Xiaobo Han ${ }^{1}$, Jing Li', Ye Tian ${ }^{1,5}$, Yajun Zhao ${ }^{1}$ and Liming Yang ${ }^{1}$

\section{Correction to: Cell Death and Disease (2016) 7: e2527 https://doi.org/10.1038/cddis.2016.433 published online 22 December 2016}

The authors wish to point out that in Fig. 1f, the picture of DAPI in the ATG5 siRNA group is incorrect. During the process of image synthesis, the authors mixed the pictures of DAPI in the control group and ATG5 siRNA group, leading to the duplicate between them of DAPI. Furthermore, the AMPK blot and the AKT blot in Fig. 2a were inadvertently duplicated with the third $\beta$-actin in Fig. 2a and AKT in Fig. 4e, respectively. The authors would like to apologize for any inconvenience this may have caused.

The correct figures are presented below.

Published online: 27 February 2019

Correspondence: Ye Tian (yetian@ems.hrbmu.edu.cn) or Yajun Zhao (zhaoyajun1964@163.com) or Liming Yang (limingyang@ems.hrbmu.edu.cn) 'Department of Pathophysiology, Key Laboratory of Cardiovascular Pathophysiology, Harbin Medical University, Harbin, China

${ }^{2}$ Department of Respiratory Medicine, The Fourth Affiliated Hospital of Harbin Medical University, Harbin, China

${ }^{3}$ Department of Cardiology, The First Affiliated Hospital, Cardiovascular

Institute, Harbin Medical University, Harbin, China

${ }^{4}$ Department of Electron Microscopic Center, Basic Medical Science College,

Harbin Medical University, Harbin, China

${ }^{5}$ Division of Cardiology, The First Affiliated Hospital, Harbin Medical University,

Harbin, China

(c) (i) Open Access This article is licensed under a Creative Commons Attribution 4.0 International License, which permits use, sharing, adaptation, distribution and reproduction c. in any medium or format, as long as you give appropriate credit to the original author(s) and the source, provide a link to the Creative Commons license, and indicate if changes were made. The images or other third party material in this article are included in the article's Creative Commons license, unless indicated otherwise in a credit line to the material. If material is not included in the article's Creative Commons license and your intended use is not permitted by statutory regulation or exceeds the permitted use, you will need to obtain permission directly from the copyright holder. To view a copy of this license, visit http://creativecommons.org/licenses/by/4.0/. 


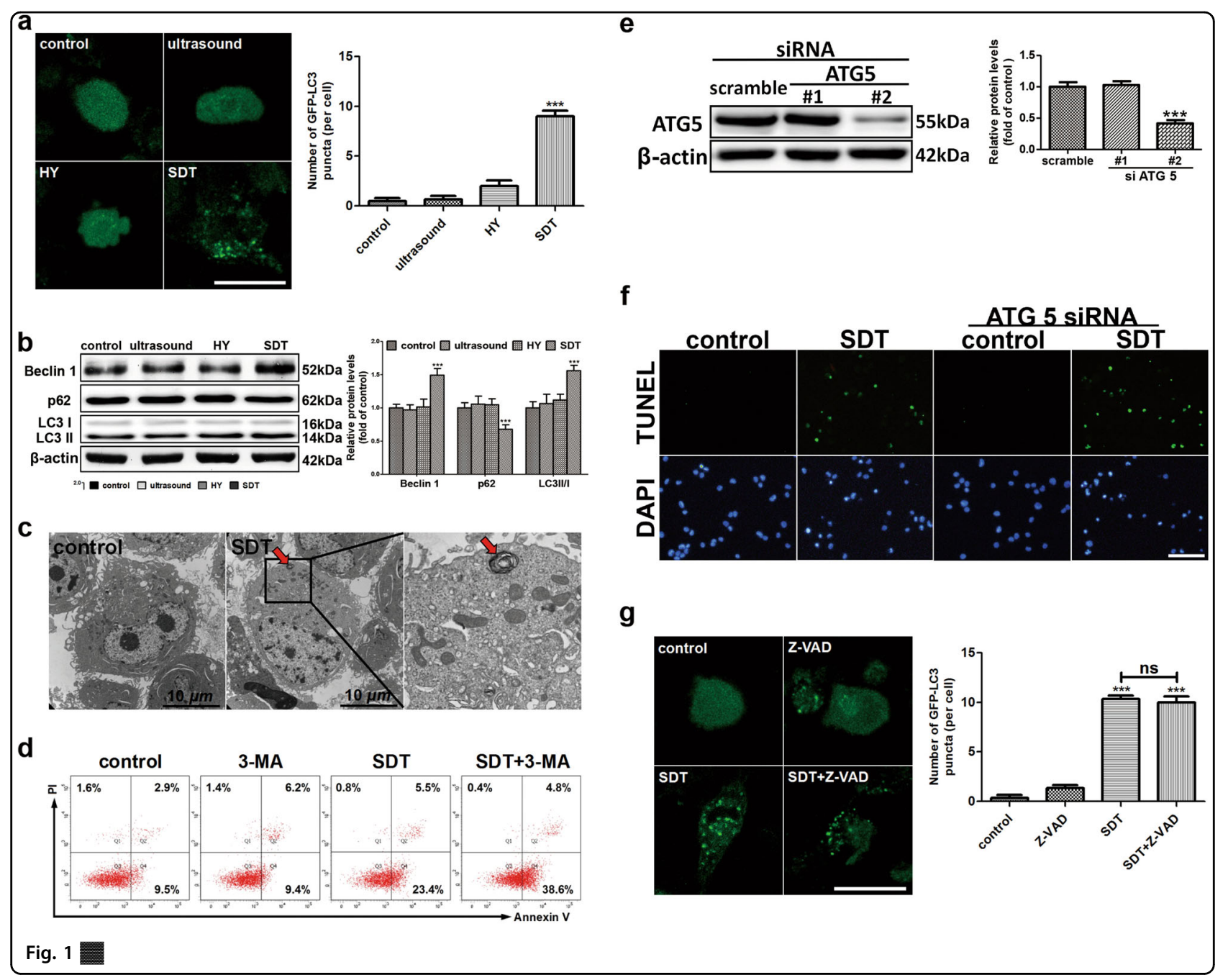




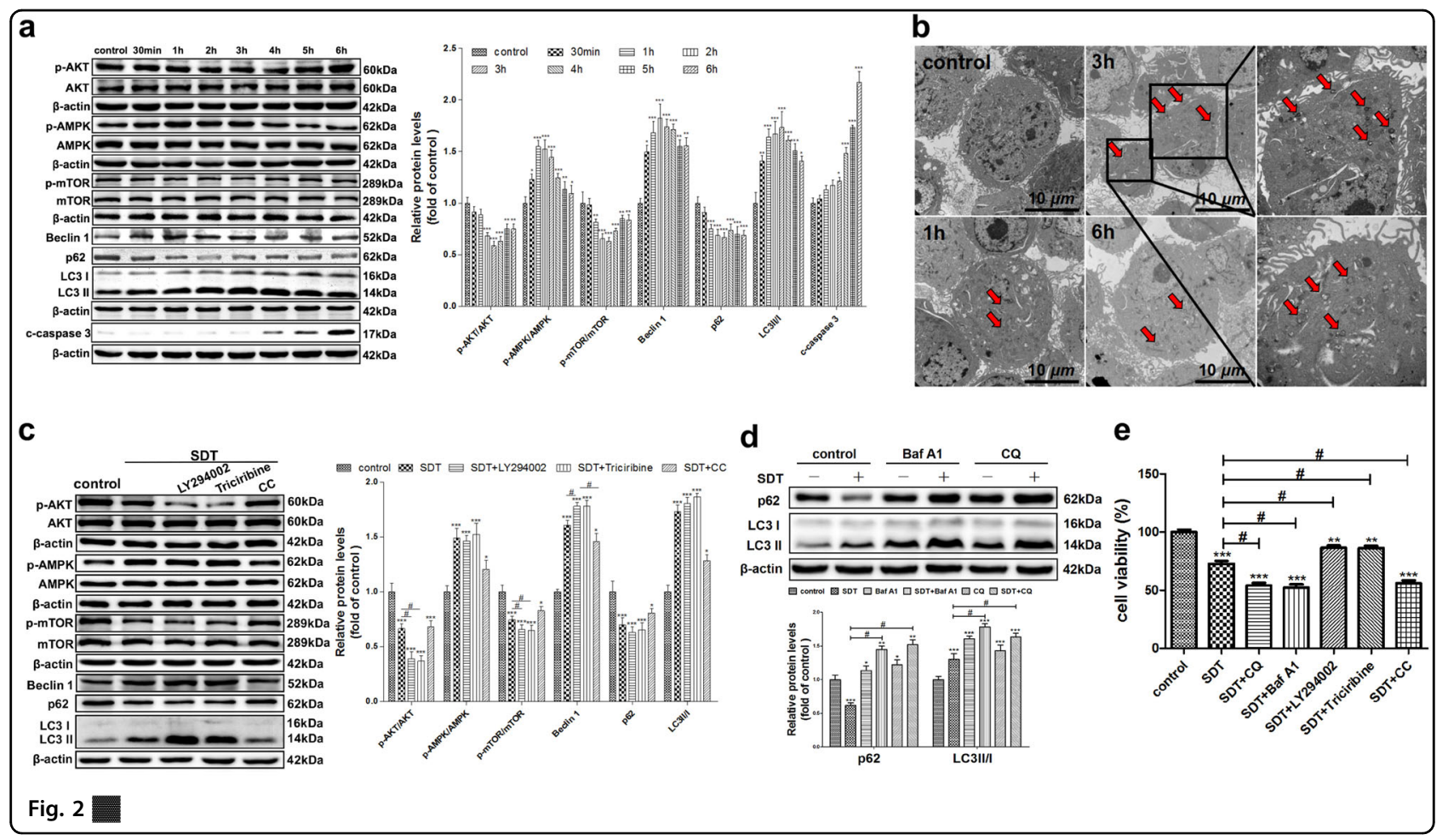

